\title{
Planning for prompt follow-up of gravitational wave events
}

\author{
Peter Shawhan*t \\ Physics Department and Joint Space-Science Institute \\ The University of Maryland, USA \\ E-mail: pshawhan@umd.edu
}

\section{Carlo Ferrigno}

ISDC Data Centre for Astrophysics

Université de Genève, Switzerland

E-mail: carlo.ferrigno@unige.ch

\begin{abstract}
The search for a gravitational wave $(\mathrm{GW})$ signal is one of the most fascinating and challenging areas of modern physics. The Advanced LIGO detectors will have a first "science run" in late 2015, while Advanced Virgo will join the network in 2016. Longer-term operations and full sensitivities should be achieved a few years later. An electromagnetic (EM) counterpart to a GW event is expected in many cases and a follow-up program is being defined to enhance the probability of firmly detecting GWs and determining their astrophysical origin. Initially, triggers resulting from the interferometer data analysis will only be distributed to partners who have signed agreements with LIGO and Virgo. Many partner groups have signed up to participate, including a group within INTEGRAL, comprising the ISDC and the project scientist. We will review the LIGO-Virgo program for electromagnetic follow-up of GW triggers and the role to be played by large-field-of-view gamma-ray instruments.
\end{abstract}

10th INTEGRAL Workshop: "A Synergistic View of the High Energy Sky" - Integral2014, 15-19 September 2014

Annapolis, MD, USA

\footnotetext{
${ }^{*}$ Speaker.

${ }^{\dagger}$ Representing the LIGO Scientific Collaboration and the Virgo Collaboration.
} 


\section{Introduction}

The general theory of relativity predicts the existence of gravitational waves, which are oscillating distortions of the spacetime metric that propagate at the speed of light. Gravitational wave $(\mathrm{GW})$ transients may be produced by massive astrophysical objects which are accelerating or changing shape, such as a binary system of neutron stars or black holes in a close orbit, or the non-axisymmetric collapse of a massive star [1]. Traveling away from the source, the GW has the form of a dimensionless tensor strain (fractional spacetime distortion), alternately stretching and squeezing the metric distance between locally inertial points in space. GW observations promise a new way to observe and understand energetic astrophysical events. However, transient GW sources are relatively rare and the GW strain amplitude decreases with distance; at the Earth it is typically of order $10^{-21}$ or smaller and thus extremely challenging to detect. (Quasiperiodic and broadband stochastic GWs can also be produced and detected with even smaller amplitudes by integrating over weeks or months of data.)

In spite of the tiny signal amplitudes, the direct detection of gravitational waves is technically feasible. Kilometer-scale laser interferometer detectors on the Earth are able to target GW signals with frequencies between about $10 \mathrm{~Hz}$ and $5 \mathrm{kHz}$, while pulsar timing array campaigns [2] and space-based detectors [3] are sensitive to lower frequencies. First-generation ground-based gravitational wave detectors were successfully constructed and operated in the past decade, led by LIGO [4] and Virgo [5]. These detectors are currently undergoing major upgrades to improve their sensitivities by an order of magnitude [6,7], and will be joined in the future by the KAGRA detector in Japan [8] and an additional LIGO observatory in India [9].

Much can be learned about physics, astrophysics and cosmology from gravitational waves by themselves and in combination with standard electromagnetic (EM) astronomy observations [10]. The GW emission comes directly out of the central engine and is not obscured or scattered by intervening material; thus GW observations complement photon diagnostics of photosphere, outflows, circumburst medium, and astronomical context that EM observations can supply. In fact, all anticipated GW transient sources are highly energetic astrophysical events, and must be relatively close (in cosmological terms) to be detectable by LIGO and Virgo. For example, the Advanced LIGO GW detectors will be able to detect binary neutron star mergers out to distances of around $200 \mathrm{Mpc}$ [11]. Some EM transients, such as gamma-ray bursts (GRBs), are routinely observed at much greater distances, but are believed to be beamed emission from a relativistic jet. On the other hand, GW emissions are only weakly beamed, and GW detectors are only weakly directional; thus the GW detectors monitor the whole sky for sources with all inclinations, and may detect closer events that would be missed by EM transient surveys, or else detected but not recognized as significant. These considerations motivate planning for joint observations, and in particular, preparing to follow up GW events promptly with EM observations by ground-based and orbiting instruments. We describe the ongoing preparations in this article.

\section{Goals of the EM follow-up program}

The EM follow-up concept is illustrated in Figure 1. The main processing task is to transfer and analyze the GW data immediately after it is recorded and identify apparent GW signals, or 


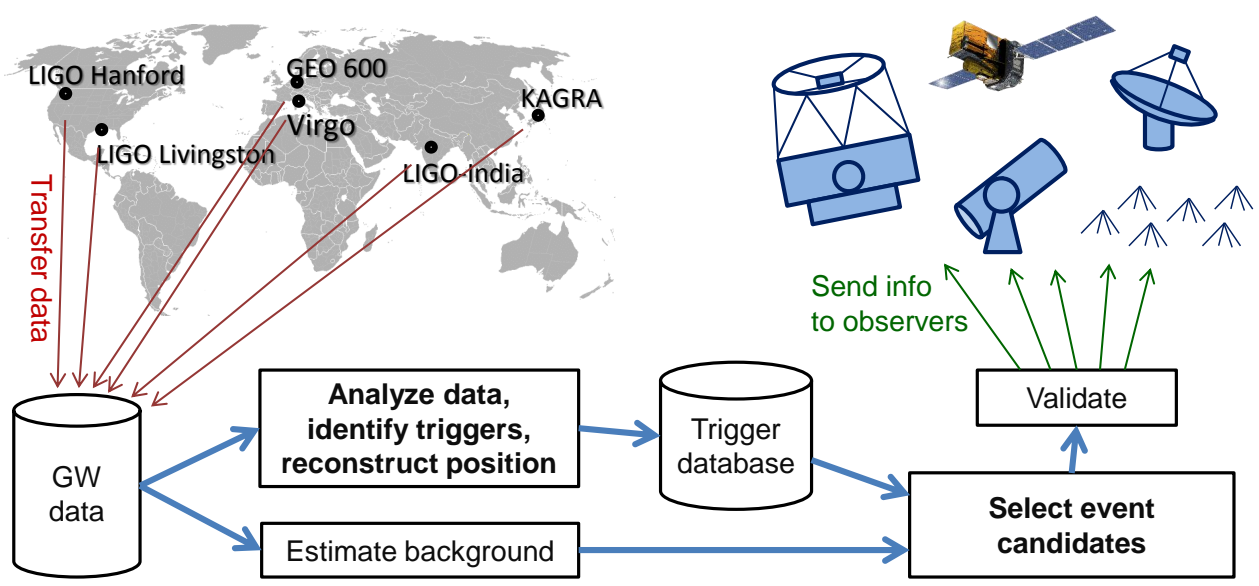

Figure 1: Main steps in processing data from the GW detector network and rapidly generating alerts for follow-up observations. (INTEGRAL image credit: ESA)

triggers. For each trigger, basic event parameters and a sky position probability map ("sky map") are reconstructed using the time-series data streams from the multiple GW detectors. The statistical significance of the trigger is evaluated by comparing its strength to the distribution of "background" triggers constructed by applying the same analysis to data streams which have been time-shifted, thus sampling the random combinations of detector noise which may mimic GW signals. In this way, the strength of a trigger can be expressed non-parametrically as the false alarm rate (FAR) for background outliers with equal or greater value of the relevant detection statistic (e.g., signalto-noise ratio). A stronger trigger has a lower lower FAR, and if the FAR is below some chosen threshold, the trigger is selected as an event candidate. After a final validation of the data quality, the event candidate information — including basic properties, estimated false alarm rate, and sky map-is sent to EM observers.

One goal of this effort is to identify GW event candidates as quickly as possible so that other observers can look for rapidly fading counterparts in various EM bands. We expect to send out alerts with latencies of order 10 minutes; the exact latency will depend on what validation checks are considered necessary, and may be longer, at least at first. For some counterpart searches, this rapid response will be crucial; on the other hand, other plausible counterparts such as kilonovae $[12,13,14]$ have slower light curves that do not even peak until some time later, so searches for those can utilize strategically scheduled observations. Aside from triggering target-of-opportunity observations, GW candidates can simply be correlated with data from ongoing transient surveys to find apparent or possible counterparts, and to prioritize further follow-up resources (e.g., spectroscopy) accordingly. This may allow the observers to identify and characterize an interesting transient, confirm a GW event candidate that may have been marginal by itself, and obtain multiwavelength (and multi-messenger) data for such events. A major challenge for identifying counterparts, however, is that the GW reconstructed sky map regions are large - typically several tens to hundreds of square degrees for the next several years. Later, when KAGRA and LIGO-India join the detector network with comparable sensitivity, they will supply more triangulation baselines and detector orientations with which to pin down the properties of a passing GW signal, including its 
sky position.

In parallel with the EM follow-up effort, LIGO/Virgo will also be monitoring GCN [24] and possibly other transient event feeds and will do deeper analysis of the GW data around the times and sky positions of notable reported events, as has been done in the past [15].

\section{First implementation}

A first version of the EM follow-up program was carried out during the latter part of the LIGO-Virgo science run in 2009-2010 [16, 17]. Data from the three GW detector sites was calibrated and transferred to the LIGO computing facility at Caltech within $\sim 1$ minute. Whenever all three detectors were operating (so that any detected signal could be localized relatively well), the data was analyzed using two low-latency search pipelines. One pipeline searched for GW signals from compact binary coalescence ( $\mathrm{CBC}$ ) events, using optimal matched filtering with a bank of $\mathrm{CBC}$ signal templates. The search focused on binaries containing at least one neutron star, since the leading models for EM counterparts involve the disruption of the neutron star(s), and used the relative arrival times at the different detectors to triangulate the apparent position of the source, supplemented by an amplitude comparison to resolve the twofold degeneracy [17]. The other pipeline searched for GW "bursts"- transients with arbitrary waveform—using a robust "coherent excess power" search algorithm (Coherent WaveBurst [18]) that was sensitive to essentially any signal in the LIGO-Virgo frequency band with duration up to $\sim 1 \mathrm{~s}$, and reconstructed the sky position probability map with a fully coherent analysis.

Triggers from either pipeline were generated with a typical latency of 3 to 6 minutes. However, the validation process for event candidates involved a checklist with many manual steps, and typically took 10 to 30 minutes. For GW event candidates which passed the validation step, the sky map was compared to a catalog of nearby galaxies (within $50 \mathrm{Mpc}$, roughly the maximum plausible distance of a detectable CBC source in that science run) to select telescope fields containing the most promising possible hosts; EM observing partners were then asked to obtain images of those fields as soon as possible, and on subsequent nights.

Ten observer groups were involved in promptly following up GW candidates during the 20092010 science run, including users of optical telescopes around the globe (ROTSE, TAROT, QUEST, PTF, SkyMapper, Pi of the Sky, Zadko, and Liverpool), the LOFAR Transients Key Science Project, and members of the Swift mission team [16]. During the ten weeks when the follow-up program was active, nine GW event candidates were followed up by at least one telescope, including two by Swift. The GW candidates were marginal by themselves and no convincing transient counterpart was found in any of the collected images, but the analysis methods developed and exercised in the process $[19,20]$ are a foundation on which to build.

\section{Preparing for the era of advanced GW detectors}

\subsection{Technical preparations}

At the time of this writing, installation of the Advanced LIGO instrumentation is complete and the detectors are being commissioned; for instance, optical components are being aligned, feedback systems are being engaged and tuned, and noise sources are being hunted down. In fact, 
the early commissioning of the LIGO Livingston detector proceeded more quickly than expected; it achieved full interferometer lock on May 26, 2014, and since then has often run for several hours at a time. The laser power is still being kept much lower than the Advanced LIGO design, but even so, the sensitivity of the detector (evaluated as the range to which a binary neutron star CBC event would be detected with $\mathrm{SNR}=8$ ) soon exceeded the best that the initial LIGO detectors were able to manage. The LIGO Hanford detector is (intentionally) being commissioned on a somewhat later schedule, but it is expected that both LIGO detectors will be ready to conduct a first (short) observing run in mid to late 2015, consistent with the schedule projected in 2013 [11]. Advanced Virgo installation and commissioning are proceeding well, and it is expected to join LIGO for an observing run in 2016. KAGRA and LIGO-India will join the GW detector network some years later $[8,11]$.

The low-latency GW data analysis is evolving compared to the 2009-10 follow-up program. The same types of GW transients (CBC and bursts) will be searched for, but the analysis pipelines have been re-implemented to improve coordinate reconstruction, speed and robustness. The burst search software (Coherent WaveBurst) uses the same basic analysis but with some incremental improvements to separate signal from glitches better and to improve the reconstructed sky map accuracy. Multiple low-latency $\mathrm{CBC}$ search pipelines are now available and are being cross-compared. Significantly, sky position reconstruction for $\mathrm{CBC}$ events now uses a low-latency Bayesian coherent algorithm which incorporates the phase and amplitude information from the different detectors [21]. Sky maps will also be refined later with full parameter estimation using MCMC methods, which are slower but potentially more precise. Typical two- and three-detector sky maps expected from the first two years of Advanced LIGO and Virgo observing runs are characterized in [21].

For the Advanced LIGO-Virgo era, the low-latency searches will be run whenever at least two GW detectors are collecting good data, and event candidate alerts will be generated. Two detectors cannot localize an event as well as three or more can, but can still indicate the time of the candidate and its possible localization along a ring in the sky, determined by the relative arrival time at the two detectors along with the antenna responses and Bayesian prior for the source population. That large area may be challenging to follow up with small field-of-view instruments, but will still be useful for correlating with other surveys or very-wide-field instruments.

Work is also in progress to re-implement the software used to select event candidates and issue alerts [22]. We hope to automate data quality checks and avoid or at least minimize the manual checks that were the dominant contribution to the latency of alerts in 2009-10.

\subsection{Organizational of the follow-up effort}

The first direct detection of gravitational waves will be a momentous occasion and must be established with care. Confident detection of the first few GW signals will require time to fully validate the data and analysis before announcing it to the public; during that time it will be important to avoid misinformation and rumors. That is part of the reason that LIGO and Virgo data has an initial proprietary period. On the other hand, LIGO and Virgo recognize that prompt follow-up observations can be extremely valuable even for the very first GW signals, and are committeed to coordinating with other observers by promptly sharing the time, significance, sky map, and basic signal properties with observers who agree to respect the confidentiality of the data. To this end, LIGO and Virgo consulted with many members of the transient astronomy community and issued 
two invitations to participate in this program [23]. To date, over 50 groups have signed memoranda of understanding (MOUs) with LIGO and Virgo to receive event candidate alerts, which will be assembled in VOEvent format [25] and distributed using the GCN system [26] to a controlled list of recipients. The participating groups represent a very broad spectrum of transient astronomy researchers and facilities, some of which are already surveying for transients and others which will be employed specifically to follow up GW event candidates. Swift, INTEGRAL, Fermi, XMMNewton, Chandra, and HXMT are some of the gamma-ray and X-ray facilities planned to be used for follow-up.

Although the event information should not be made public, all participants within the program ("inside the bubble") are encouraged to communicate freely with each other and share their observing plans and findings. Observers may also coordinate directly, to the extent they want. We hope that this will enable a high efficiency for finding and characterizing counterparts to GW events.

LIGO and Virgo have promised that further in the future-specifically, after $4 \mathrm{GW}$ events have been published-high-confidence GW events will be released promptly to the public. Lowerconfidence event candidates, that are not sure detections without the corroborating evidence of an EM counterpart or a statistical analysis of an ensemble, will continue to be shared with partners who have signed MOUs.

\subsection{Observing strategies}

We have begun discussions of science strategies, logistical details and testing plans with observers. One thing which has become clear is that different observers will target different potential counterparts to GW transients, such as short GRBs (whether initially identified by gamma-ray missions or not), "orphan afterglows" in X-rays or optical emission (perhaps from an "off-axis" binary merger, for which the jet is not aligned with the line of sight), kilonovae, kilonova precursors [27], radio afterglows of binary mergers, core-collapse supernovae, fast radio bursts, etc.

Different plausible counterparts call for various approaches in terms of the timing of observations and strategies to deal with the large sky regions reconstructed for the GW candidates. Instruments with large field of view will have a natural advantage; a few, like INTEGRAL's 30-by30-degree IBIS imager [28], or the proposed ISS-Lobster focusing X-ray imager [29], are actually well matched to the typical size of LIGO-Virgo error regions. Instruments with smaller fields of view could tile the error region, though that may require a very large amount of observing time. One strategy which can help is to target only nearby galaxies within the error region, though that is only appropriate for seeking extragalactic sources expected to be within or near galaxies, of course. Alternatively, some observers may work together to divide up the GW sky map error region. Even with such strategies, there may be multiple possible counterparts, and the challenge will be to confidently identify the true counterpart. High-energy signatures, multi-band light curves, and spectroscopy may provide crucial evidence.

\section{INTEGRAL plans for GRBs and GW transients}

An electromagnetic counterpart is expected to be associated with at least some Advanced LIGO/Virgo triggers, and telescopes with large field of view and sensitivity in the X- to gammaray domain are probably in the best position to detect the counterpart [30]. INTEGRAL data are 
scanned in near real time to search for GRBs, and can also be examined specially following a GW event candidate alert. Even if the GW detectors supply only the time of event, with little or no constraint on sky position (for example, during the 2015 commissioning phase), a temporal coincidence of events will be useful; and in future years the spatial error box will be significantly reduced.

A central role for transient searches is taken by the INTEGRAL Science Data Centre (ISDC) in collaboration with the INTEGRAL Burst Alert System (IBAS) team [31]. We will exploit procedures derived from the IBAS system, which can be optimised to the particular data set. As a routine task, ISDC scans the observations performed by INTEGRAL for quality check and detection of transient phenomena. There are well established procedures to determine the significance of hard $\mathrm{X}$-ray and gamma-ray events detected with the instruments on board INTEGRAL, which will be used in this study [31,32], and to guarantee a localization accuracy of a few arc minutes. From this activity, one GRB is detected in the IBIS field of view every 1-2 months. ISDC also scans the publicly available signal from the anti-coincidence shield of the SPI instrument on board INTEGRAL, which detects about two gamma-ray bursts per week, albeit without localization capability. When these events are confirmed by other satellites in the InterPlanetary Network (IPN [33]), they are localized using triangulation. Gamma-ray events discovered serendipitously during these routine activities will be advertised to the astrophysical community with GCN or Astronomer's Telegrams, following well-established procedures.

For seeking counterparts to GW event candidates, due to the importance of such events, the details concerning data access will be handled by the INTEGRAL Project Scientist. A team will perform an in-depth analysis of INTEGRAL data in a \pm 1 day window centered on the time of the trigger from the GW detectors. This will allow us to detect any possible gamma-ray signal associated with the GW trigger, including precursors or delayed emissions, which might last several hours in the case of magnetar flares (e.g., [34]). Such a window is essential because, for the dithering strategy, a source can fall outside the field of view of the imager at the time of trigger and only be pointed at earlier or later. Coincidence with GW triggers will be disclosed only after careful checks with the collaboration, following the guidelines of the MOU, and publication will involve agreement from a large consortium in case both GW and EM channels are involved. A possible causal relation with GW events should be evaluated for each event based on the characteristics of the possible counterpart, the possible phenomenon originating the signal, and the detection time scale.

\section{Summary}

Gravitational-wave transient events are expected to be produced by sources which generate detectable electromagnetic emissions too, at least some fraction of the time. The GW signature will complement photon diagnostics of the photosphere, outflows, circumburst medium, and/or the astronomical context of the event as revealed by EM observations. The first complete implementation of a follow-up program using LIGO and Virgo event candidates was tested in 2009-10. We are now preparing to provide rapid alerts to observers in the Advanced GW detector era. The alerts to be issued will enable correlation with transient surveys as well as prompt and delayed targeted follow-up observations. Many observers, including members of the INTEGRAL science team, are 
currently planning how to receive and act on GW event candidate alerts. Coordinated observations will commence with the first Advanced LIGO observing run in the second half of 2015, and will improve over time as the GW detectors reach full sensitivity and the detector network is enlarged.

\section{Acknowledgments}

P.S. gratefully acknowledges the support of the U.S. National Science Foundation through grants PHY-1068549 and PHY-1404121.

The LIGO Scientific Collaboration and Virgo Collaboration gratefully acknowledge the support of the United States National Science Foundation (NSF) for the construction and operation of the LIGO Laboratory, the Science and Technology Facilities Council (STFC) of the United Kingdom, the Max-Planck-Society (MPS), and the State of Niedersachsen/Germany for support of the construction and operation of the GEO600 detector, the Italian Istituto Nazionale di Fisica Nucleare (INFN) and the French Centre National de la Recherche Scientifique (CNRS) for the construction and operation of the Virgo detector and the creation and support of the EGO consortium. The authors also gratefully acknowledge research support from these agencies as well as by the Australian Research Council, the International Science Linkages program of the Commonwealth of Australia, the Council of Scientific and Industrial Research of India, Department of Science and Technology, India, Science \& Engineering Research Board (SERB), India, Ministry of Human Resource Development, India, the Spanish Ministerio de Economía y Competitividad, the Conselleria d'Economia i Competitivitat and Conselleria d'Educació, Cultura i Universitats of the Govern de les Illes Balears, the Foundation for Fundamental Research on Matter supported by the Netherlands Organisation for Scientific Research, the Polish Ministry of Science and Higher Education, the FOCUS Programme of Foundation for Polish Science, the European Union, the Royal Society, the Scottish Funding Council, the Scottish Universities Physics Alliance, the National Aeronautics and Space Administration, the Hungarian Scientific Research Fund (OTKA), the Lyon Institute of Origins (LIO), the National Research Foundation of Korea, Industry Canada and the Province of Ontario through the Ministry of Economic Development and Innovation, the National Science and Engineering Research Council Canada, the Brazilian Ministry of Science, Technology, and Innovation, the Carnegie Trust, the Leverhulme Trust, the David and Lucile Packard Foundation, the Research Corporation, and the Alfred P. Sloan Foundation. The authors gratefully acknowledge the support of the NSF, STFC, MPS, INFN, CNRS and the State of Niedersachsen/Germany for provision of computational resources.

This document has been assigned the identifier LIGO-P1400248-v1.

\section{References}

[1] K. Riles, Gravitational waves: Sources, detectors and searches, Progress in Particle \& Nuclear Physics 68 (2013) 1 [arXiv:1209.0667].

[2] R. N. Manchester (for the IPTA), The International Pulsar Timing Array, Classical and Quantum Gravity 30 (2013) 224010 [arXiv: 1309 . 7392].

[3] P. Amaro-Seoane et al., eLISA: Astrophysics and cosmology in the millihertz regime, GW Notes 6 (2013) 4 [arXiv:1201.3621]. 
[4] B. P. Abbott et al., LIGO: the Laser Interferometer Gravitational-Wave Observatory, Reports on Progress in Physics 72 (2009) 076901 [arXiv: 0711.3041 ].

[5] T. Accadia et al., Virgo: A laser interferometer to detect gravitational waves, J. of Instrumentation 7 (2012) P03012.

[6] The LIGO Scientific Collaboration, Advanced LIGO, arXiv: 1411.4547.

[7] F. Acernese et al., Advanced Virgo: A 2nd generation interferometric gravitational wave detector, Classical and Quantum Gravity 32 (2015) 024001 [arXiv: 1408 .3978].

[8] Y. Aso, Y. Michimura, K. Somiya, M. Ando, O. Miyakawa, T. Sekiguchi, D. Tatsumi, and H. Yamamoto, Interferometer design of the KAGRA gravitational wave detector, PRD 88 (2013) 043007 [arXiv:1306.6747].

[9] C. S. Unniktrishnan, IndIGO and LIGO-India scope and plans for gravitational wave research and precision metrology in India, IJMPD 22 (2013) 1341010.

[10] B. S. Sathyaprakash and B. F. Schutz, Physics, astrophysics and cosmology with gravitational waves, Living Reviews in Relativity 12 (2009) 2, http://www.livingreviews.org/lrr-2009-2.

[11] J. Aasi et al. (LIGO Scientific Collaboration and Virgo Collaboration), Prospects for localization of gravitational wave transients by the Advanced LIGO and Advanced Virgo observatories, arXiv: 1304.0670.

[12] B. D. Metzger, G. Martínez-Pinedo, S. Darbha, E. Quataert, A. Arcones, D. Kasen, R. Thomas, P. Nugent, I. V. Panov, and N. T. Zinner, Electromagnetic counterparts of compact object mergers powered by the radioactive decay of r-process nuclei, MNRAS 406 (2010) 2650 [arXiv:1001.5029].

[13] J. Barnes and D. Kasen, Effect of a high opacity on the light curves of radioactively powered transients from compact object mergers, ApJ 775 (2013) 19 [arXiv: 1303.5787].

[14] D. Grossman, O. Korobkin, S. Rosswog, and T. Piran, The long-term evolution of neutron star merger remnants - II. Radioactively powered transients, MNRAS 439 (2014) 757 [arXiv: 1307.2943 ].

[15] J. Abadie et al., Search for gravitational waves associated with gamma-ray bursts during LIGO science run 6 and Virgo science runs 2 and 3, ApJ 760 (2012) 12 [arXiv: 1205.2216 ].

[16] J. Abadie et al., Implementation and testing of the first prompt search for gravitational wave transients with electromagnetic counterparts, A\&A 539 (2012) A124 [arXiv: 1109 . 3498].

[17] J. Abadie et al., First low-latency LIGO+Virgo search for binary inspirals and their electromagnetic counterparts, A\&A 541 (2012) A155 [arXiv:1112.6005].

[18] S. Klimenko, I. Yakushin, A. Mercer, and G. Mitselmakher, A coherent method for detection of gravitational wave bursts, Classical and Quantum Gravity 25 (2008) 114029 [arXiv: 0802 . 3232].

[19] J. Aasi et al., First searches for optical counterparts to gravitational-wave candidate events, ApJS 211 (2014) 7 [arXiv:1310.2314].

[20] P. A. Evans et al., Swift follow-up observations of candidate gravitational-wave transient events, ApJS 203 (2012) 28 [arXiv: 1205.1124$].$

[21] L. P. Singer, L. R. Price, B. Farr, A. L. Urban, C. Pankow, S. Vitale, J. Veitch, W. M. Farr, C. Hanna, K. Cannon, T. Downes, P. Graff, C.-J. Haster, I. Mandel, T. Sidery, and A. Vecchio, The first two years of electromagnetic follow-up with Advanced LIGO and Virgo, ApJ 795 (2014) 105 [arXiv: 1404 .5623]. 
[22] P. S. Shawhan (for the LIGO Scientific Collaboration and Virgo Collaboration), Rapid alerts for following up gravitational wave event candidates, in Proc. SPIE 8448, Observatory Operations: Strategies, Processes, and Systems IV (2012) 84480Q.

[23] http://www.ligo.org/scientists/GWEMalerts.php

[24] http://gcn.gsfc.nasa.gov/gen_describe.html

[25] R. D. Williams and R. L. Seaman, Transient event notification with VOEvent, in Astronomical Society of the Pacific Conference Series 382 (2007) 425.

[26] S. Barthelmy, GCN and VOEvent: A status report, Astronomische Nachrichten 329 (2008) 340.

[27] B. D. Metzger, A. Bauswein, S. Goriely, and D. Kasen, Neutron-powered precursors of kilonovae, MNRAS in press, arXiv:1409.0544.

[28] P. Ubertini et al., IBIS: The Imager on-board INTEGRAL, A\&A 411 (2003) 131.

[29] J. Camp, S. D. Barthelmy, L. Blackburn, K. Carpenter, N. Gehrels, J. Kanner, F. E. Marshall, J. L. Racusin, and T. Sakamoto, Using ISS telescopes for electromagnetic follow-up of gravitational wave detections of NS-NS and NS-BH mergers, Exp. Astron. 36 (2013) 505 [arXiv : 1304.3705 ].

[30] B. D. Metzger and E. Berger, What is the most promising electromagnetic counterpart of a neutron star binary merger?, ApJ 746 (2012) 48 [arXiv: 1108 . 6056].

[31] S. Mereghetti, D. Götz, J. Borkowski, R. Walter, and H. Pedersen, The INTEGRAL Burst Alert System, A\&A 411 (2003) L291.

[32] V. Savchenko, A. Neronov, and T. J.-L. Courvoisier, Timing properties of gamma-ray bursts detected by SPI-ACS detector onboard INTEGRAL, A\&A 541 (2012) A122.

[33] K. Hurley, T. Cline, I. Mitrofanov, E. Mazets, S. Golenetskii, F. Frontera, E. Montanari, C. Guidorzi, and M. Feroci, The Current Performance of the Third Interplanetary Network, AIP Conf Proc 662 (2003) 473 [arXiv: astro-ph/0201374].

[34] V. Savchenko, A. Neronov, V. Beckmann, N. Produit, and R. Walter, SGR-like flaring activity of the anomalous $X$-ray pulsar $1 E$ 1547.0-5408, A\&A $\mathbf{5 1 0}$ (2010) A77. 